Revue de droit comparé du travail et de la sécurité sociale

3 | 2018

La participation des travailleurs dans la grande entreprise privée et publique

\title{
Le système de workfare en Belgique temporairement freiné par la Cour constitutionnelle
}

\section{Vanessa De Greef}

\section{(Q) OpenEdition}

\section{Journals}

Édition électronique

URL : https://journals.openedition.org/rdctss/1926

DOI : $10.4000 /$ rdctss. 1926

ISSN : 2262-9815

Éditeur

Centre de droit comparé du travail et de la sécurité sociale

Édition imprimée

Date de publication : 1 novembre 2018

Pagination : 160-165

ISSN : 2117-4350

Référence électronique

Vanessa De Greef, « Le système de workfare en Belgique temporairement freiné par la Cour constitutionnelle », Revue de droit comparé du travail et de la sécurité sociale [En ligne], 3 | 2018, mis en ligne le 01 novembre 2021, consulté le 12 novembre 2021. URL : http://journals.openedition.org/ rdctss/1926; DOI : https://doi.org/10.4000/rdctss.1926

\section{(c) $($ ) $\odot$ EY}

Revue de droit comparé du travail et de la sécurité sociale est mise à disposition selon les termes de la Licence Creative Commons Attribution - Pas d'Utilisation Commerciale - Pas de Modification 4.0 International. 


\section{LE SYSTÈME DE WORKFARE EN BELGIQUE TEMPORAIREMENT FREINÉ PAR LA COUR CONSTITUTIONNELLE}

En juillet 2016, le législateur fédéral belge a adopté un système de workfare dans le domaine du droit à l'intégration sociale (équivalent belge du RSA). Deux années plus tard, la Cour constitutionnelle annule le dispositif au motif qu'il contrevient au système de répartition des compétences. Si un tel dispositif peut donc être prochainement rétabli par une des autorités compétentes, la Cour a également soulevé des éléments qui ne seront pas facilement solubles pour les rédacteurs du prochain projet de réforme. La présente note a pour but de retracer brièvement le contexte et la réception du dispositif en Belgique (I) et d'analyser l'arrêt de la Cour constitutionnelle (II)'.

\section{I - CONTEXTE ET RÉCEPTION DU DISPOSITIF DE WORKFARE EN BELGIQUE}

Le 21 juillet 2016, le législateur fédéral belge instaure un dispositif de workfare dans le domaine du droit à l'intégration sociale. Par workfare, nous désignons les programmes qui requièrent des allocataires sociaux qu'ils travaillent gratuitement en échange de prestations sociales. En Belgique, ce nouveau dispositif doit être mis en étroite relation avec le phénomène de contractualisation du droit à l'intégration sociale. En effet, le système de workfare belge est inséré dans un contrat, le projet individualisé d'intégration sociale (PIIS). Le législateur fédéral belge a établi que « le projet individualisé d'intégration sociale peut avoir trait à un service communautaire, qui en fait alors partie intégrante. Le service communautaire consiste à exercer des activités sur une base volontaire qui constituent une contribution positive tant pour le parcours de développement personnel de l'intéressé que pour la société $»^{2}$.

1 Cour const., arrêt n 86/2018 du 5 juillet 2018.

2 Article 11, §1 er, al. 5 de la loi du 21 juillet 2016 modifiant la loi du 26 mai 2002 concernant le droit à l'intégration sociale (Moniteur belge, 2 août 2016). Voir également l'arrêté d'exécution : I'arrêté royal du 3 octobre 2016 modifiant l'arrêté royal du 11 juillet 2002 portant règlement général en matière de droit à l'intégration sociale, (Moniteur belge, 11 octobre 2016) et la circulaire du 12 octobre 2016 relative à la loi du 21 juillet 2016 modifiant la loi du 26 mai 2002 concernant le droit à l'intégration sociale (non publiée au Moniteur belge). 


\section{BelgiQue}

Juridiquement, le bénéficiaire peut donc être amené à signer ce contrat et doit ensuite l'exécuter, entre autres en vue de démontrer sa disposition à travailler qui est une condition d'octroi du droit à l'intégration sociale. Le non-respect du projet individualisé peut entraîner la suspension temporaire partielle ou totale du revenu d'intégration.

Sans surprises, le nouveau dispositif a été fort critiqué par le terrain, comme le révèlent ces slogans: " pas de salaire comme pour un véritable emploi, pas de liberté comme pour un véritable bénévolat » ${ }^{3}$, " une société qui exploite les plus pauvres et les plus précaires? Un système qui détruit des emplois, en les remplaçant par du travail gratuit forcé? Nous nous y opposons! $»^{4}$. Le nouveau dispositif de workfare souleva également plusieurs questions auprès des experts et acteurs de terrain : le service communautaire peut-il être volontaire alors qu'il fait partie du contrat que signe le bénéficiaire et dans lequel il s'engage à exécuter une série d'obligations afin de recevoir son revenu d'intégration ? Sans référence explicite à la loi du 3 juillet 2005 relative aux droits des volontaires ${ }^{5}$, cette loi belge trouvet-elle à s'appliquer? Si ce service n'est pas vraiment volontaire, ne se trouve-t-on pas dans les conditions constitutives du travail forcéb ? En effet, la jurisprudence internationale exige de ne pas se limiter au consentement formel et d'examiner la validité du consentement exprimé, lequel peut avoir été contraint par l'Etat. Comment s'assurer que ce service va constituer une contribution positive tant pour le parcours de développement personnel de l'intéressé que pour la société ? La question est importante car la justification d'un objectif d'intérêt général par l'Etat est analysée dans le cadre du test de proportionnalité que la Cour européenne des droits de l'homme (CEDH) met en œuvre lorsqu'elle apprécie la réalité du consentement et l'existence d'un travail forcé?.

Ces différents questionnements ont été « coulés » en moyens juridiques dans le cadre d'un recours introduit par la Ligue des droits de l'Homme belge (section francophone) et l'association "Collectif Solidarité contre l'Exclusion: Emploi et Revenus pour tous». D'autres associations ont également envoyé plusieurs mémoires à la Cour constitutionnelle afin de soutenir le recours.

3 Y. Martens, "Service communautaire » : travail gratuit (et forcé) pour tous les allocataires ? " Revue Politique, 30 juillet 2018.

4 Extrait de la communication du Réseau wallon de lutte contre la pauvreté.

5 Moniteur belge, 29 août 2005.

6 Ou a minima face à une violation d'une des autres composantes du droit au travail librement entrepris.

7 C.E.D.H., Van der Mussele c. Belgique, 23 novembre 1983, § 38. Voir également, E. Dermine, "Activation Policies for the Unemployed and The International Case Law on the Prohibition of Forced Labour », Journal européen des droits de l'homme/European Journal of Human Rights, vol. 1, $n^{\circ} 5,2013$, pp. 746-776 ; E. Dermine et V. De Greef, « Le droit au travail librement entrepris (art. 1er, § 2 de la CSE) face aux situations de travail non protégées par le droit social. Les cas du travail pénitentiaire et des mesures de workfare ", Actualités des droits sociaux fondamentaux, sous la dir. de S. Van Drooghenbroeck, F. Dorssemont et G. Van Limberghen, Bruxelles, La Charte, 2016, p. 315 à 317. 
Parmi les autres moyens avancés dans la requête, il y avait entre autres le fait que le service communautaire est un travail qui ne respecte ni les règles relatives au contrat de travail, ni celles applicables aux prestations de travail effectuées sous l'autorité d'une autre personne. Un moyen moins idéologiquement marqué que celui relatif au droit social ou aux droits économiques, sociaux et culturels a trait au fait que les dispositions instaurant le service communautaire ont été adoptées par le législateur fédéral alors qu'elles auraient dû l'être par le législateur régional, seul compétent depuis la sixième réforme de l'Etat (2014) en matière de «mise au travail des personnes qui bénéficient du droit à l'intégration sociale ou du droit à l'aide sociale financière $»^{8}$.

Enfin, un moyen plus surprenant mobilisé par ces associations concerne les libertés de circulation et le respect du jeu de la concurrence garantis par le TFUE. Aux yeux des requérants, l'entrave que constitue l'apparition sur le marché du service communautaire crée une discrimination entre, d'une part, les entreprises ou particuliers qui souhaitent prester des services en Belgique qui ne souffrent pas de la concurrence avec les personnes employées dans le cadre du service communautaire et, d'autre part, les entreprises ou particuliers qui veulent travailler dans des domaines où elles souffrent de cette concurrence.

\section{II - ANALYSE DE L'ARRÊT DE LA COUR CONSTITUTIONNELLE}

Sans avoir répondu à tous les moyens, l'arrêt de la Cour constitutionnelle apporte des précisions à plusieurs titres. Tout d'abord, l'arrêt annule les dispositions qui instaurent le service communautaire parce que "le service communautaire relève de la compétence attribuée aux régions " depuis que la sixième réforme de l'Etat a transféré aux régions le soin de prendre en charge "la mise au travail des personnes qui bénéficient du droit à I'intégration sociale $»^{9}$. Certes, les travaux préparatoires de la sixième réforme de l'Etat avaient précisé qu'il s'agissait de transférer de l'autorité fédérale vers les régions « les programmes d'accompagnement visant à réinsérer les bénéficiaires d'un revenu d'intégration sur le marché du travail » mais les exemples de programmes mentionnés ne concernaient pas le service communautaire et ce, assez logiquement vu qu'il n'existait pas ${ }^{10}$. Malgré cela, il n'était toutefois pas acquis que la Cour reconnaisse le service communautaire comme tel. Certes, le législateur fédéral avait précisé dans les travaux préparatoires que ce service doit " être dirigé vers un futur travail rémunéré » mais la Cour aurait pu avoir une lecture plus restrictive des programmes auxquels il était fait référence ${ }^{11}$.

8 Article $6, \S 1^{\mathrm{er}}, \mathrm{IX}, 2 / 1^{\circ}$ de la loi spéciale du 8 août 1980 de réformes institutionnelles modifié par l'article 22 de la loi spéciale du 6 janvier 2014 relative à la sixième réforme de l'Etat (Moniteur belge, 31 janvier 2014).

9 Cour const., arrêt n 86/2018 du 5 juillet 2018, §B.30.1.

10 Doc. Parl., Sénat, 25 juillet 2013, 5-2232/1, p. 13.

11 Doc. Parl., Chambre, 27 mai 2016, 54-1864/001, p. 6. 


\section{BelgiQue}

La Cour a ensuite développé plus en avant son raisonnement. Elle précise que le demandeur du droit à l'intégration sociale peut refuser la proposition d'accepter un service communautaire mais que son acceptation lui permet également de prouver sa disposition à travailler qui est une condition d'octroi du droit à l'intégration sociale ${ }^{12}$. Une fois accepté, le service devient par ailleurs contraignant pour le bénéficiaire. La Cour précise que le défaut d'accomplissement des prestations prévues peut entraîner "une conséquence grave " pour le bénéficiaire, que ce service ne peut être considéré comme une activité "sans obligation » et qu'il n'entre donc pas dans le cadre légal relatif aux droits des volontaires ${ }^{13}$. Sans répondre au moyen relatif au fait que le service communautaire est un travail qui doit se voir appliquer la loi sur le contrat de travail ${ }^{14}$, la Cour précise cependant que « le service communautaire présente des caractéristiques proches de celles d'un travail rémunéré » et que «les activités pouvant faire l'objet d'un service communautaire qui doivent constituer une contribution positive pour la société, ne peuvent être distinguées, entoutes circonstances et par nature, des activités pouvant faire l'objet d'un travail rémunéré. Rien n'interdit en effet que les activités pouvant être accomplies au titre du service communautaire ne puissent également, si les moyens financiers sont disponibles, faire l'objet d'une rémunération ${ }^{15}$. Il découle de cette partie de l'arrêt, empreinte de réalisme et de pragmatisme, que si un des prochains législateurs régionaux assume que le service communautaire n'est pas un travail bénévole, il devra se justifier à plusieurs titres : il devra établir non seulement en quoi ledit service se distingue d'un travail qui devrait se voir appliquer la législation relative au droit du travail mais il devra aussi veiller à ne pas porter atteinte au droit au travail librement entrepris et, entre autres, au fait qu'un tel service ne peut être constitutif de travail forcé.

Enfin, la Cour s'est prononcée au sujet d'une nouvelle disposition relative à l'extension $d u$ caractère obligatoire $d u$ projet individualisé d'intégration sociale à tout nouveau bénéficiaire du droit à l'intégration sociale ${ }^{16}$. Jusqu'alors, un tel projet n'était obligatoire qu'à l'égard des jeunes de moins de 25 ans. Cette mesure constitue, avec l'instauration du service communautaire, l'autre nouveauté de la réforme de 2016. A cet égard, les requérants ont insisté sur le défaut d'encadrement législatif des obligations prévues dans le contrat signé avec le bénéficiaire : il s'agit, d'après eux, d'une délégation de pouvoir contraire au principe de légalité en matière de droits fondamentaux (en l'espèce le droit au respect de la vie privée et le droit à l'aide sociale). Les requérants ont également fait valoir qu'une absence de cadre législatif est susceptible de créer des discriminations entre allocataires

12 Cour const., arrêt n 86/2018 du 5 juillet 2018, §B.28.1.

13 Cour const., arrêt n 86/2018 du 5 juillet 2018, §B.29.1.

14 Loi du 3 juillet 1978 relative aux contrats de travail, Moniteur belge, 22 août 1978.

15 Ibidem.

16 Article 11, §2, c) de la loi du 26 mai 2002 sur le droit à l'intégration sociale (Moniteur belge, 2 août 2016). 
sociaux en fonction de la politique adoptée par le centre public d'action sociale dont ils dépendent. Enfin, ils ont soulevé que l'extension de ce contrat signifie un renforcement du caractère conditionnel du droit à l'intégration sociale et par là, une violation de l'obligation de standstill (équivalent belge du principe de non-régression) contenue à l'article 23 de la Constitution.

La Cour n'a pas accueilli favorablement ces différents moyens : primo, la loi contient assez d'éléments permettant de déterminer le projet individualisé d'intégration sociale de façon à ne pas donner lieu à des atteintes disproportionnées au droit au respect de la vie privée des demandeurs ou au droit à l'aide sociale. Le législateur fédéral n'a donc pas créé une indétermination contraire au principe de légalité. Elle a cependant mentionné - ce qui est, à notre estime, en décalage avec la réalité sociale des bénéficiaires concernés souvent éloignés d'un accès effectif à la justice - que «si de telles atteintes devaient se produire, elles pourraient être sanctionnées par les juridictions compétentes " $^{17}$. Deuxio, elle a rejeté l'argument de la discrimination relative aux pratiques divergentes selon les centres publics d'action sociale compétents en rappelant que le projet individualisé est un outil de travail social qui, par définition, doit être adapté à la situation de chaque personne. II en résulte forcément des différences qui ne se heurtent au principe d'égalité et de non-discrimination que si elles entraînent une atteinte disproportionnée aux droits fondamentaux ${ }^{18}$. La Cour s'appuie alors sur les travaux préparatoires pour réfuter pareille atteinte : le demandeur peut toujours se faire accompagner d'un tiers et s'adresser au tribunal du travail ${ }^{19}$. Qu'il nous soit permis, à nouveau, de douter du manque de faisabilité pratique pour des personnes précarisées et souvent très solitaires. Tertio, la Cour balaye le moyen relatif à la violation de l'obligation de standstill en refusant d'examiner si la mesure constitue un recul significatif du niveau de protection conféré au droit fondamental à l'aide sociale pour les bénéficiaires du droit à l'intégration sociale. Elle se limite à constater que la mesure est " justifiée par un motif d'intérêt général, à savoir favoriser l'intégration professionnelle et sociale des personnes» ${ }^{20}$. A ce sujet, il est regrettable que la Cour ne procède pas, comme elle le fait parfois, à un réel contrôle de proportionnalité pour analyser l'atteinte potentielle à l'obligation de standstil/21.

17 Cour const., arrêt n ${ }^{\circ} 86 / 2018$ du 5 juillet 2018, §B.9.5.

18 Cour const., arrêt nº 86/2018 du 5 juillet 2018, §B.17.2.

19 Cour const., arrêt nº 86/2018 du 5 juillet 2018, §B.17.3.

20 Cour const., arrêt n $86 / 2018$ du 5 juillet 2018, §B.21.2.

21 Sur l'obligation de standstill, voir I. Hachez, Le principe de standstill dans le droit des droits fondamentaux : une irréversibilité relative, Bruxelles/Athènes/ Baden-Baden, Bruylant / Sakkoulas / Nomos Verlagsgesellschaft, 2008. 


\section{BelgiQue}

Dans les mois à venir, il est probable qu'un des législateurs régionaux - et probablement en priorité le législateur flamand qui est désireux d'enclencher davantage de dispositifs d'activation dite « sociale »- adopte une forme de service communautaire. II est également attendu, si les partenaires fédéral et régionaux parviennent à trouver un accord, qu'un tel dispositif pénètre le domaine de l'assurance chômage conformément à l'accord de gouvernement fédéral 2014-2019 qui prévoit que le chômeur devrait à l'avenir travailler gratuitement dans le cadre d'un service dit « communautaire »22. Enfin, un arrêt du Conseil d'Etat doit encore être rendu au sujet de l'arrêté d'exécution de la loi instaurant le service communautaire et le projet individualisé d'intégration sociale. Pour les dispositions qui sont encore d'application après l'arrêt de la Cour, l'arrêt du Conseil d'Etat pourrait éclairer les zones d'ombre de l'arrêt de la Cour constitutionnelle.

22 Doc. parl., Chambre, Déclaration de politique gouvernementale, 14 octobre 2014, n 0020/001, p. 28-29. 